\title{
KPC-mediated resistance in Klebsiella pneumoniae in two hospitals in Padua, Italy, June 2009-December 2011: massive spreading of a KPC-3-encoding plasmid and involvement of non-intensive care units
}

Sara N Richter ${ }^{1,2^{*}}$, Ilaria Frasson ${ }^{1}$, Elisa Franchin ${ }^{1,2}$, Cristina Bergo ${ }^{2}$, Enrico Lavezzo ${ }^{1}$, Luisa Barzon ${ }^{1,2}$, Antonietta Cavallaro ${ }^{2}$ and Giorgio Palü ${ }^{1,2}$

\begin{abstract}
Background: Klebsiella pneumoniae carbapenemases (KPCs) producing bacteria have emerged as a cause of multidrug-resistant nosocomial infections worldwide. KPCs are plasmid-encoded enzymes capable of hydrolysing a broad spectrum of beta-lactams, including carbapenems and monobactams, therefore worryingly limiting antimicrobial treatment options. Analysis of circulating bacterial strains and KPC alleles may help understanding the route of KPC dissemination and therefore help containing the infection.

Methods: KPC-producing Klebsiella pneumoniae dissemination in two 1580- and 300- bed hospitals in Padua, Italy, from initial outbreak in 2009 to late 2011 was analysed. Molecular and clinical epidemiology, including bacterial strains, KPC-encoding plasmid sequences and associated resistance genes, involved hospital wards and relocation of patients were described. Routine antimicrobial susceptibility testing and MIC of carbapenems on clinical isolates were performed. Detection of resistance genes was obtained by PCR and sequencing. MLST, PFGE and ERIC were used for molecular genotyping. Plasmid analysis was obtained by digestion with restriction enzymes and deep sequencing.

Results: KPC-positive clinical samples were isolated from nearly 200 patients. In the initial outbreak intensive care units were almost exclusively involved, while medical, surgical and long-term wards were successively massively concerned. Analysis of KPC alleles, plasmids and bacterial sequence types (STs) indicated that during the initial outbreak KPC-3 in ST258 and KPC-2 in ST147 were each confined in one of the two surveilled hospitals. While KPC-2 dissemination was effectively contained, KPC-3 in ST258 cross-spreading was observed. The simultaneous presence of two carbapenemases, VIM-1 and KPC-2, in the same isolate was also observed in three patients. Total sequencing of plasmid content of two KPC-3 strains showed novel association of resistance plasmids.

(Continued on next page)
\end{abstract}

\footnotetext{
* Correspondence: sara.richter@unipd.it

'Department of Molecular Medicine, University of Padua, Via Gabelli, 63 35121, Padua, Italy

${ }^{2}$ Azienda Ospedaliera di Padova, Microbiology and Virology Unit, via Giustiniani 2, 35121, Padua, Italy
}

\section{Ciomed Central}

(c) 2012 Richter et al.; licensee BioMed Central Ltd. This is an Open Access article distributed under the terms of the Creative Commons Attribution License (http://creativecommons.org/licenses/by/2.0), which permits unrestricted use, distribution, and reproduction in any medium, provided the original work is properly cited. 
(Continued from previous page)

Conclusions: The acquired molecular epidemiology demonstrated that 1) both acquisitions from outward sources and patient relocation within the hospitals were responsible for the observed spreading; 2) KPC-3-encoding Klebsiella pneumoniae ST258 prevailed over other strains. In addition, the described massive transfer of KPC-mediated resistance to non-intensive care units may anticipate spreading of resistance to the non-hospitalized population. Therefore, genotypic analysis alongside phenotypic identification of carbapenemase producers, also at the carriage state, is advisable to prevent and contain further carbapenemase resistance dissemination.

Keywords: KPC, Carbapenemase, Klebsiella pneumoniae, Plasmid-mediated antimicrobial resistance, Gram-negative, Nosocomial infections

\section{Background}

Bacteria producing Klebsiella pneumoniae carbapenemases (KPCs) have emerged as a cause of multidrugresistant nosocomial infections worldwide [1]: they are capable of hydrolysing a broad spectrum of beta-lactams including penicillins, cephalosporins, carbapenems and monobactam [2], except cephamycins [3]. The emergence of carbapenem-resistant Enterobacteriaceae is particularly worrisome because carbapenems are widely regarded as the drugs of choice for the treatment of severe infections caused by extended-spectrum betalactamase (ESBL)-producing Enterobacteriaceae [4]; the additional frequent co-occurrence of genes conferring resistance to other classes of drugs has severely limited antimicrobial treatment options [1]. KPC-producing Klebsiella pneumoniae isolates were first identified in North Carolina in 1996 [5]; the first outbreak of KPCproducing $K$. pneumoniae outside the United States was described in Israel in 2006 [6]. The European country in which most cases have been reported so far is Greece, where the infection is considered endemic [7]. In Italy a rapid increase in the number of cases has been recently described [8]. However, no long-term surveillance has been reported in Italy, yet. In general, in all countries outbreaks have always been restricted to hospitals, in particular to intensive care units (ICU) [9].

KPCs are plasmid-encoded enzymes whose high mobility and dissemination are related to a Tn3-based transposon, Tn4401, which is carried by large plasmids varying in size and structure [1]. The most frequently found KPCencoding plasmid is pKpQIL [10]. To date 11 different $\mathrm{KPC}$ variants (KPC1/2-KPC12) have been described [11]. In Italy both KPC-2 and KPC-3 have been reported, with a higher prevalence of KPC-3. Many sequence types (STs) of $K$. pneumoniae have been found associated to KPC enzyme production: the most globally distributed, therefore likely the most environmentally fit, is ST258, which was found coupled to both KPC-3 and KPC-2 [12].

Here we present the first long-time follow up of KPC dissemination in Italy. KPC clinical isolates were surveilled from initial outbreak in 2009 until late 2011 in two hospitals (nearly 2000 beds) in Padua, a large town in North-East Italy counting over 936.000 inhabitants, including provincial citizens. In particular, we focussed on KPC allele dissemination, $K$. pneumoniae ST spreading and identity of $\mathrm{KPC}$-encoding plasmids with respect to the two involved hospitals. In addition, the clinical epidemiology showed worrying progressive transfer of KPC infection from intensive care units (ICUs) to surgical, medical and long-term hospital wards.

\section{Results}

Starting in 2009, standard microbiological testing revealed a dramatic rise in the number of $K$. pneumoniae isolates with increased MIC of imipenem $(\geq 2 \mathrm{mg} / \mathrm{L})$ in the intensive-care units (ICU) of the Teaching Hospital ( $\mathrm{TH}$, one ward) and Saint Antony Hospital (SAH, one ward), two distinct hospital complexes in the city of Padua, offering 1580 and 300 beds, respectively. In particular, in ICU/TH and ICU/SAH, isolates with imipenem MIC $\geq 2 \mathrm{mg} / \mathrm{L}$ were absent until June 2009, increased from $17 \%$ and $12 \%$, respectively, in July-December 2009, to $87 \%$ and 54\%, respectively, in January-June 2010. From June 2009, we started to search for KPC genes on clinical isolates exhibiting imipenem $\mathrm{MIC} \geq 2 \mathrm{mg} / \mathrm{L}$, within all units of the two hospitals. In addition, since summer 2010 a systematic search for KPC-positive isolates was performed twice a week by rectal swabbing of all patients from $\mathrm{ICU} / \mathrm{TH}$.

Molecular and clinical epidemiology Up to June 2010, 29 patients with at least one KPC-positive K. pneumoniae isolate were identified: as shown in Figure 1, in both hospitals the vast majority (66\% and $100 \%)$ was from ICUs. Remarkably, two different KPC genes in two K. pneumoniae sequence types (STs) were prevalent in the two hospitals: in particular, KPC-3 in ST258 (83\%) had spread in $\mathrm{TH}$, while KPC-2 in ST147 (82\%) was prevalently found in SAH. KPC-3-positive isolates belonging to the two new ST527 and ST37 were also detected in TH. Since MSLT takes into account modifications in highly conserved genes hence revealing mainly long-term changes, two additional molecular typing techniques, ERIC-PCR and PFGE, were used to detect short-time alterations. In 


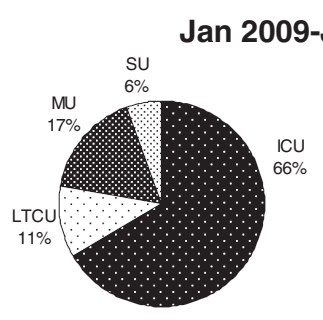

$\mathrm{TH}$

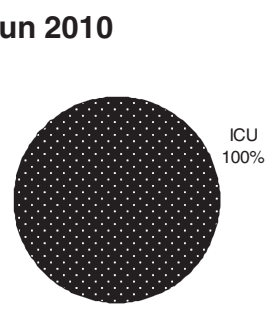

$\mathrm{SAH}$

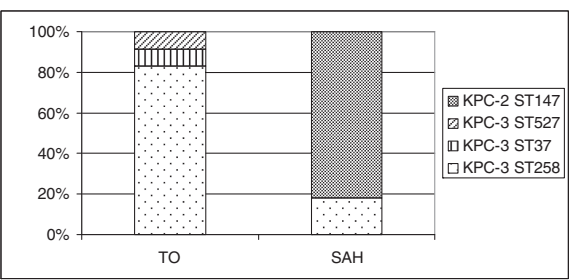

Jul 2010-Dec 2011
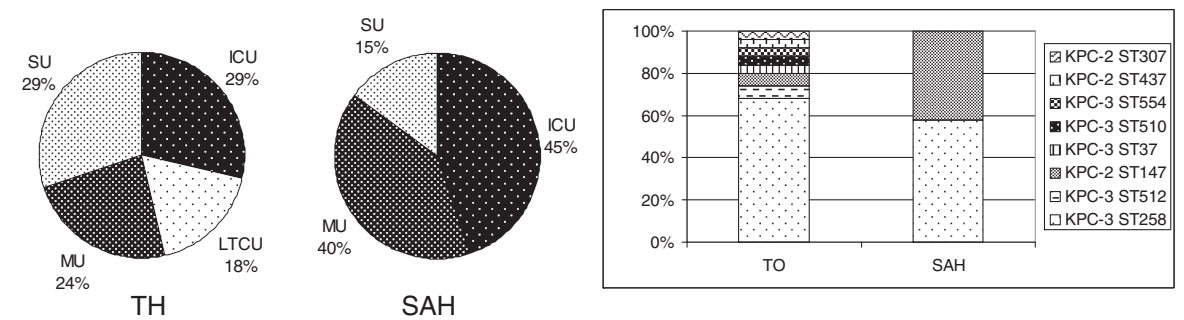

Figure 1 Epidemiology of KPC genes in two hospitals (TH and SAH). On the left, pie charts show the evolution of KPC spreading in hospital wards from January 2009 to Jun 2010 and from July 2010 to December 2011. On the right, bar graphs indicate prevalence of KPC genes and their association with K. pneumoniae STs during the two considered periods of time. Abbreviations are: ICU = Intensive Care Unit; $M U=M e d i c a l$ Unit; SU = Surgical Unit; LTCU = Long Term Care Unit; TH=Teaching Hospital; SAH = Saint Antony Hospital.

particular, ERIC-PCR identifies changes in variable regions and PFGE in the whole genome. All clinical isolates were classified into 4 PFGE profiles, represented in Figure 2a, which matched the classification made by MLST. Three profiles were identified with ERIC-PCR (Figure 2b): these matched the MLST and PFGE but for one sample (\#7), indicating a less discriminatory power of this technique or possibly a closer genetic relatedness between this sample (encoding KPC-3 in ST37 with PFGE profile D) and samples encoding KPC-3 in ST258 with PFGE profile B. Overall these data indicate that KPCpositive samples derived from four different $K$. pneumoniae strains with poor genetic relatedness. Genotypic and phenotypic properties of these KPC-positive isolates are reported in Table 1.

Carbapenem resistance coupled to KPC production was followed up from July 2010 to December 2011. A total of 160 different patients with at least one isolate were found. Of these, 140 patients were in TH. Remarkably, in this time period in both hospitals KPC-positive isolates were mainly detected in non-ICUs, such as surgical, medical and long-term care wards (Figure 1).

Molecular analysis showed that in the second period of analysis both KPC-2 and KPC-3 had moved within the two hospitals: in particular, KPC-3-positive isolates belonging to ST258 were mostly represented in both nosocomia (Figure 1). Concurrently, several novel STs, such as ST512, ST745 (two new single locus variants of ST258, in gapA and rpoB, respectively), ST510, ST554 associated with KPC-3, and ST307, ST437 with KPC-2 were detected.

To understand the involvement of non-ICUs after the initial outbreak, relocation of patients was studied in the two periods of time (Jan 2009-Jun 2010 and Jul 2010-Dec 2011) (Figure 3). In the first period even though most of the patients were initially admitted to non-ICUs, the vast majority of multi-drug-resistant (MDR) isolates, including KPC-positive bacteria, were found during the following ICU admission (76\%) and were mostly maintained in subsequent post-ICUs, where the percentage of new acquisitions was $24 \%$. In the second period, the number of new acquisitions of MDR organisms (MDRO) dramatically raised in non-ICUs (6\% and $38 \%$ in pre-ICU and post-ICU admission, respectively), while new acquisitions in ICU decreased. Noteworthy, the percentage of patients with newly and already acquired MDR isolates was very high with respect to the total number of KPC-positive patients hospitalized in ICUs, both in the first and second period (96\% and $83 \%$, respectively), and was in general maintained in post-ICUs ( $67 \%$ and $63 \%$, respectively).

To slow down KPC spread, starting from 2011, one ICU has been devoted to patients colonized/infected by KPC organisms. In addition, most non-ICUs introduced the policy of isolating KPC-positive patients in a dedicated room, with restricted access to hospital staff and relatives.

Plasmid analysis To verify the similarity of KPCpositive plasmids, plasmids from representative samples 


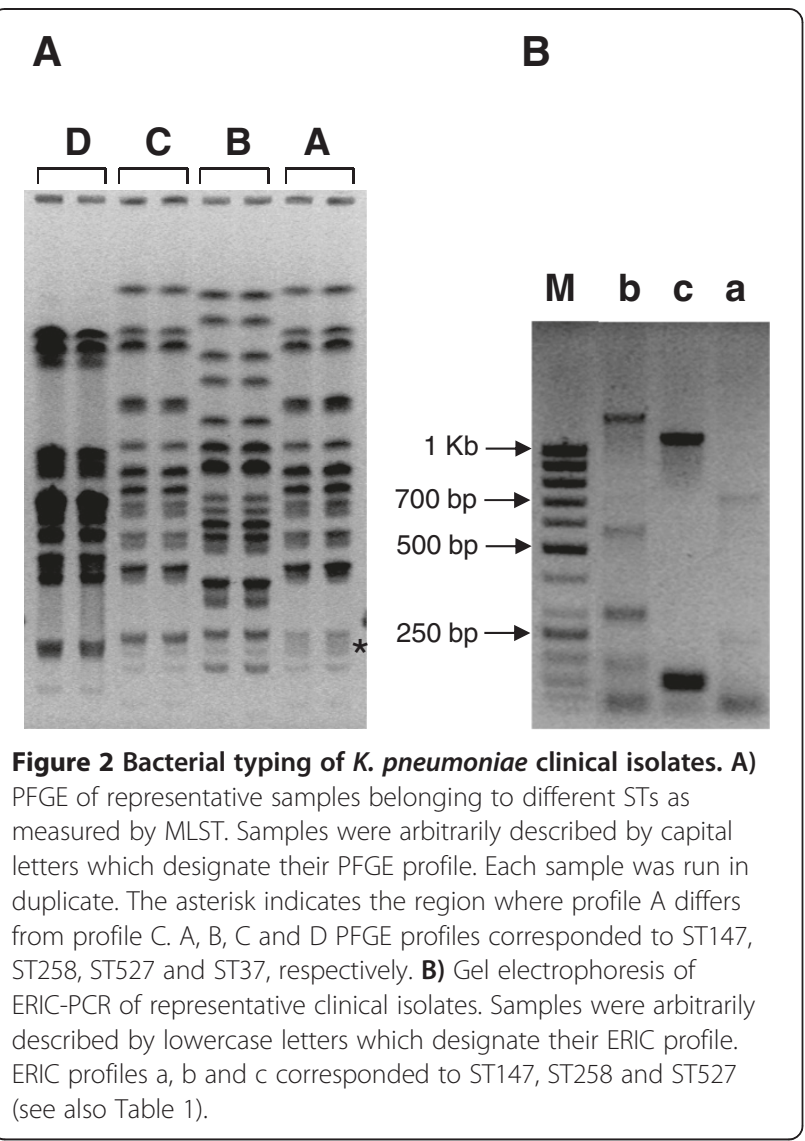

from each identified ST were transformed into E. coli Top10, purified, digested with restriction enzymes and run on agarose gel. Additionally, plasmids from ST258, ST147 and ST37 detected in November 2009 were compared to those collected in September 2011 to check for plasmid variation within the same ST. As shown in Figure 4, no variation was found between plasmids collected in 2009 and 2011 (see ST258, ST37, ST147). In addition, KPC-3-encoding plasmids from ST258, ST37 and ST527 displayed identical digestion profiles, but differed from that from ST512. KPC-2-positive plasmids from ST147, ST307 and ST437 exhibited a unique digestion pattern.

Partial plasmid sequencing revealed that $b l a_{\mathrm{KPC}}$ genes were in all cases embedded in a Tn4410-like transposon. The KPC-3-encoding plasmids present in K. pneumoniae ST258 (identical to the plasmid contained in ST37 and ST147) and ST512 were further subjected to deep sequencing. We found that both KPC-3 plasmid sequences matched that of $\mathrm{pKpQIL}$, which has been previously reported in K. pneumoniae ST258 [10]. K. pneumoniae ST512 additionally contained a second plasmid identical to the recently described plasmid pIncX-SHV [13], which explains the above depicted partially different digestion profile.
Phenotypic and molecular analysis of additional resistance determinants The presence of KPC-encoding plasmids increased the MIC of imipenem and meropenem from 0.25 and $0.32 \mathrm{mg} / \mathrm{L}$, respectively, to $1.5-3$ and $0.38-1 \mathrm{mg} / \mathrm{L}$, respectively. In addition, transformed Top10 cells gained resistance to $\beta$-lactams, due to blaTEM-1 and $b l a_{\text {OXA-9 }}$ genes present in all transformed plasmids, while remained susceptible to sulfamethoxazole/trimethoprim, aminoglycosides and tigecycline, indicating that KPC-plasmids did not contain additional resistance genes to these classes of antibiotics.

Other chromosomal resistance genes or genes located on different plasmids were present in the clinical isolates. Antibiotic susceptibility testing showed that isolates were multiresistant and retained susceptibility only to tigecycline and colistin. Molecular analysis showed the presence of $b l a_{\mathrm{SHV}-12}(58 \%)$ and $b l a_{\mathrm{SHV}-11}(50 \%)$ in KPC-2 and KPC-3-positive strains, respectively. Noteworthy, the latter association (i.e KPC-3 and bla $a_{\mathrm{SHV}-11}$ ) has only been reported in Northern Europe and USA $[14,15]$. Most of the isolates were positive for $b l a_{\mathrm{TEM}-1}$ (86\%), bla $a_{\text {СтX-M-15 }}(2.5 \%)$, and bla $a_{\text {OXA-9 }}(88 \%)$. Interestingly, $4 \mathrm{KPC}-2$ isolates were additionally positive for the metallo- $\beta$-lactamase $b l a_{\mathrm{VIM}-1}$, association which has been reported only in Greece and very recently in Germany $[16,17]$. In our clinical setting, there was no evidence of extensive spreading of $b l a_{\mathrm{VIM}-1}$ in other species.

\section{Discussion and conclusions}

Analysis of KPC-mediated resistance in two hospitals in Padua, Italy, revealed two distinct spreading behaviours: during initial outbreak, ICUs were almost exclusively involved. Each of the two identified $b l a_{\mathrm{KPC}}$ genes identified was very clearly confined in only one of the two hospitals. Four $K$. pneumoniae STs were identified: ST258 associated with both KPC-2 and KPC-3 has been reported worldwide [1], and in particular in Italy [8]; ST147 associated with KPC-2 has been detected in Greece [7], while ST37 and ST527 have never been identified associated with a bla $a_{\text {KPC }}$ gene. These 4 STs differed for at least $5 / 7$ loci, hence had poor genetic relatedness, indicating nosocomial acquisition of bla $a_{\mathrm{KPC}}$ both by outward sources (e.g. patients that travelled abroad or were moved from other hospitals) and by horizontal transfer of KPC-encoding plasmids. KPC spreading was additionally favoured by clonal expansion of KPC-positive ST258 and ST147, as attested by prevalence of these STs in each of the two hospitals.

A strikingly different behaviour occurred in the second period of analysis: KPC-mediated resistance massively moved from ICUs to medical, surgical and long-term care wards. Additionally, KPC-3-positive ST258 prevailed over KPC-2 ST147, and many novel STs, which have never been reported associated with KPC, were 
Table 1 Properties of KPC-positive clinical isolates and transformant strains. Isolates were collected from 29 patients in the time period January 2009-June 2010

\begin{tabular}{|c|c|c|c|c|c|c|c|c|c|c|c|c|c|c|c|c|c|c|c|}
\hline \multirow[t]{2}{*}{$\begin{array}{l}\text { Sample } \\
\text { ID }\end{array}$} & \multirow[t]{2}{*}{$\begin{array}{c}\text { Hospital } \\
\text { unit }\end{array}$} & \multicolumn{2}{|c|}{ carbapenemase } & \multicolumn{4}{|c|}{$\beta$-lactamase } & \multicolumn{3}{|c|}{$\begin{array}{c}\text { molecular } \\
\text { typing }\end{array}$} & \multicolumn{9}{|c|}{ MIC (mg/L) } \\
\hline & & KPC & VIM & TEM & SHV & CTX & OXA & MLST & PFGE & ERIC & IPM & MER & CTX & CAZ & FEP & SXT & GEN & AMK & TGC \\
\hline 13 & SAH-ICU & 2 & 1 & 1 & 12 & nd & 9 & 147 & $A$ & $a$ & $\geq 16$ & $\geq 16$ & $\geq 64$ & $\geq 64$ & $\geq 64$ & $\geq 320$ & 4 & 32 & 2 \\
\hline 20 & $\mathrm{SAH}-\mathrm{ICU}$ & 2 & 1 & 1 & 12 & nd & 9 & 147 & A & a & $\geq 16$ & $\geq 16$ & $\geq 64$ & $\geq 64$ & $\geq 64$ & $\geq 320$ & 4 & $\geq 64$ & 2 \\
\hline 39 & SAH-ICU & 2 & nd & 1 & nd & nd & 9 & 147 & A & a & $\geq 16$ & $\geq 16$ & $\geq 64$ & $\geq 64$ & $\geq 64$ & 40 & $\leq 1$ & $\leq 2$ & 2 \\
\hline 40 & $\mathrm{SAH}-\mathrm{ICU}$ & 2 & nd & 1 & nd & nd & 9 & 147 & A & a & $\geq 16$ & $\geq 16$ & 32 & 16 & 32 & 40 & $\leq 1$ & $\leq 2$ & 2 \\
\hline Top10-41 & & & & & & & & & & & 8,2 & $2,0.5$ & $\geq 64$ & 4 & 2 & $\leq 20$ & $\leq 1$ & $\leq 2$ & $\leq 0.5$ \\
\hline 42 & $\mathrm{SAH}-\mathrm{ICU}$ & 2 & nd & 1 & 12 & nd & 9 & 147 & A & a & $\geq 16$ & $\geq 16$ & 32 & 16 & 32 & 40 & $\leq 1$ & $\leq 2$ & 2 \\
\hline 46 & $\mathrm{SAH}-\mathrm{ICU}$ & 2 & nd & 1 & 12 & nd & 9 & 147 & A & a & $\geq 16$ & $\geq 16$ & 32 & 16 & $\geq 64$ & 40 & $\leq 1$ & $\leq 2$ & 2 \\
\hline 49 & SAH-ICU & 2 & 1 & nd & nd & nd & 9 & 147 & A & a & $\geq 16$ & $\geq 16$ & 32 & 16 & $\geq 64$ & 40 & $\leq 1$ & $\leq 2$ & 2 \\
\hline 65 & $\mathrm{SAH}-\mathrm{ICU}$ & 3 & nd & 1 & 11 & nd & 9 & 258 & $B$ & $b$ & $\geq 16$ & 4 & 8 & $\geq 64$ & 8 & $\geq 320$ & 2 & $\geq 64$ & 2 \\
\hline 68 & $\mathrm{SAH}-\mathrm{ICU}$ & 3 & nd & 1 & 11 & nd & 9 & 258 & B & $b$ & $\geq 16$ & 4 & 32 & $\geq 64$ & 8 & $\geq 320$ & 4 & $\geq 64$ & 2 \\
\hline 69 & SAH-ICU & 2 & nd & 1 & 12 & nd & 9 & 147 & A & a & $\geq 16$ & $\geq 16$ & 32 & 16 & $\geq 64$ & 40 & $\leq 1$ & $\leq 2$ & 2 \\
\hline Top10-69 & & & & & & & & & & & 8,3 & 1,1 & $\geq 64$ & 4 & 2 & $\leq 20$ & $\leq 1$ & $\leq 2$ & $\leq 0.5$ \\
\hline 74 & SAH-ICU & 2 & nd & nd & nd & nd & nd & 147 & A & a & $\geq 16$ & $\geq 16$ & $\geq 64$ & $\geq 64$ & $\geq 64$ & $\geq 320$ & 4 & $\geq 64$ & 2 \\
\hline 35 & $\mathrm{TH}-\mathrm{ICU}$ & 3 & nd & 1 & nd & nd & 9 & 258 & B & $\mathrm{b}$ & $\geq 16$ & 8 & $\geq 64$ & $\geq 64$ & 16 & $\geq 320$ & $\geq 16$ & 8 & 2 \\
\hline Top10-35 & & & & & & & & & & & 8,2 & $1,0.75$ & $\geq 64$ & 4 & 2 & $\leq 20$ & $\leq 1$ & $\leq 2$ & $\leq 0.5$ \\
\hline 44 & $\mathrm{TH}-\mathrm{ICU}$ & 3 & nd & 1 & nd & nd & 9 & 258 & B & b & 8 & 8 & 8 & $\geq 64$ & 16 & $\geq 320$ & $\geq 16$ & 16 & 2 \\
\hline Top10-44 & & & & & & & & & & & $8,1.5$ & $1,0.5$ & $\geq 64$ & 4 & 2 & $\leq 20$ & $\leq 1$ & $\leq 2$ & $\leq 0.5$ \\
\hline 45 & $\mathrm{TH}-\mathrm{ICU}$ & 3 & nd & 1 & 11 & nd & 9 & 258 & $\mathrm{~B}$ & b & $\geq 16$ & 2 & $\geq 64$ & $\geq 64$ & 16 & $\geq 320$ & $\geq 16$ & 8 & 2 \\
\hline 48 & $\mathrm{TH}-\mathrm{ICU}$ & 3 & nd & nd & nd & nd & 9 & 258 & $\mathrm{~B}$ & $b$ & $\geq 16$ & 8 & $\geq 64$ & $\geq 64$ & 16 & $\geq 320$ & $\geq 16$ & 8 & 2 \\
\hline 53 & $\mathrm{TH}-\mathrm{ICU}$ & 3 & nd & 1 & nd & nd & 9 & 258 & B & $b$ & $\geq 16$ & 8 & $\geq 64$ & $\geq 64$ & 16 & $\geq 320$ & $\geq 16$ & $\geq 64$ & 2 \\
\hline Top10-53 & & & & & & & & & & & 8,2 & $2,0.38$ & $\geq 64$ & 4 & 2 & $\leq 20$ & $\leq 1$ & $\leq 2$ & $\leq 0.5$ \\
\hline 63 & $\mathrm{TH}-\mathrm{ICU}$ & 3 & nd & 1 & nd & nd & 9 & 258 & $B$ & $b$ & $\geq 16$ & 8 & $\geq 64$ & $\geq 64$ & 32 & $\geq 320$ & $\geq 16$ & 16 & 2 \\
\hline 71 & $\mathrm{TH}-\mathrm{ICU}$ & 3 & nd & 1 & 11 & nd & 9 & 258 & $\mathrm{~B}$ & $b$ & 4 & 2 & 16 & $\geq 64$ & 8 & $\geq 320$ & $\geq 16$ & 8 & 2 \\
\hline 72 & TH-ICU & 3 & nd & 1 & 11 & nd & 9 & 258 & $\mathrm{~B}$ & b & $\geq 16$ & 4 & 16 & $\geq 64$ & 8 & $\geq 320$ & 4 & $\geq 64$ & 4 \\
\hline 12 & TH-SU & & nd & nd & nd & nd & nd & 527 & C & c & $\geq 16$ & $\leq 0.25$ & $\leq 1$ & $\leq 1$ & $\leq 1$ & $\geq 320$ & $\leq 1$ & 4 & 2 \\
\hline Top10-12 & & & & & & & & & & & 8,2 & $2,0.5$ & $\geq 64$ & 4 & 2 & $\leq 20$ & $\leq 1$ & $\leq 2$ & $\leq 0.5$ \\
\hline 14 & $\mathrm{TH}-\mathrm{ICU}$ & 3 & nd & 1 & 11 & nd & 9 & 258 & B & b & $\geq 16$ & 8 & 8 & $\geq 64$ & 8 & $\geq 320$ & 2 & $\geq 64$ & 4 \\
\hline 19 & $\mathrm{TH}-\mathrm{ICU}$ & 3 & nd & 1 & 11 & nd & 9 & 258 & B & $b$ & $\geq 16$ & 2 & 8 & $\geq 64$ & $\geq 64$ & $\geq 320$ & 2 & $\geq 64$ & 2 \\
\hline 8 & TH-MU & 3 & nd & 1 & 11 & nd & 9 & 258 & B & b & $\geq 16$ & 2 & 8 & $\geq 64$ & 16 & $\geq 320$ & 4 & $\geq 64$ & 2 \\
\hline 17 & TH-MU & 3 & nd & 1 & 11 & nd & 9 & 258 & B & b & $\geq 16$ & 4 & 8 & $\geq 64$ & 8 & $\geq 320$ & 4 & $\geq 64$ & 4 \\
\hline 18 & TH-MU & 3 & nd & 1 & 11 & nd & 9 & 258 & $B$ & $b$ & 4 & 2 & 16 & $\geq 64$ & 8 & $\geq 320$ & 4 & $\geq 64$ & 4 \\
\hline 4 & TH-LTCU & 3 & nd & 1 & 11 & nd & 9 & 258 & B & b & 4 & 2 & 8 & $\geq 64$ & 8 & $\geq 320$ & 4 & $\geq 64$ & 2 \\
\hline 7 & TH-LTCU & 3 & nd & 1 & 11 & 15 & 9 & 37 & D & $b$ & $\geq 4$ & 2 & $\geq 64$ & $\geq 64$ & $\geq 64$ & $\geq 320$ & 8 & $\leq 2$ & 2 \\
\hline Top10-7 & & & & & & & & & & & 8,3 & 2,1 & $\geq 64$ & 4 & 2 & $\leq 20$ & $\leq 1$ & $\leq 2$ & $\leq 0.5$ \\
\hline 21 & $\mathrm{TH}-\mathrm{ICU}$ & 3 & nd & 1 & 11 & nd & 9 & 258 & B & b & $\geq 16$ & $\geq 16$ & $\geq 64$ & $\geq 64$ & $\geq 64$ & $\geq 320$ & 4 & $\geq 64$ & 1 \\
\hline 64 & $\mathrm{TH}-\mathrm{ICU}$ & 3 & nd & 1 & nd & nd & 9 & 258 & B & b & $\geq 16$ & 8 & 8 & $\geq 64$ & 16 & $\geq 320$ & $\geq 16$ & 16 & 2 \\
\hline Top10-64 & & & & & & & & & & & 8,2 & $1,0.38$ & $\geq 64$ & 4 & 2 & $\leq 20$ & $\leq 1$ & $\leq 2$ & $\leq 0.5$ \\
\hline Top10 & & & & & & & & & & & $\begin{array}{l}\leq 1 \\
0.25\end{array}$ & $\begin{array}{l}\leq 0.25 \\
0.32\end{array}$ & $\leq 1$ & $\leq 1$ & $\leq 1$ & $\leq 20$ & $\leq 1$ & $\leq 2$ & $\leq 0.5$ \\
\hline
\end{tabular}

IPM = imipenem; MER = meropenem; CTX = cefotaxime; CAZ = ceftazidime; FEP = cefepime; SXT = sulfamethoxazole-trimethoprim; GEN = gentamicin; $\mathrm{AMK}=$ amikacin; TGC = tigecycline. Transformed $E$. coli strains are indicated as Top10 and the number corresponding to the clinical sample from which the plasmid was obtained.

In transformed Top10 strains, MIC values of imipenem and meropenem were obtained by Vitek (first MIC value) and E-test (second MIC value in italics). 

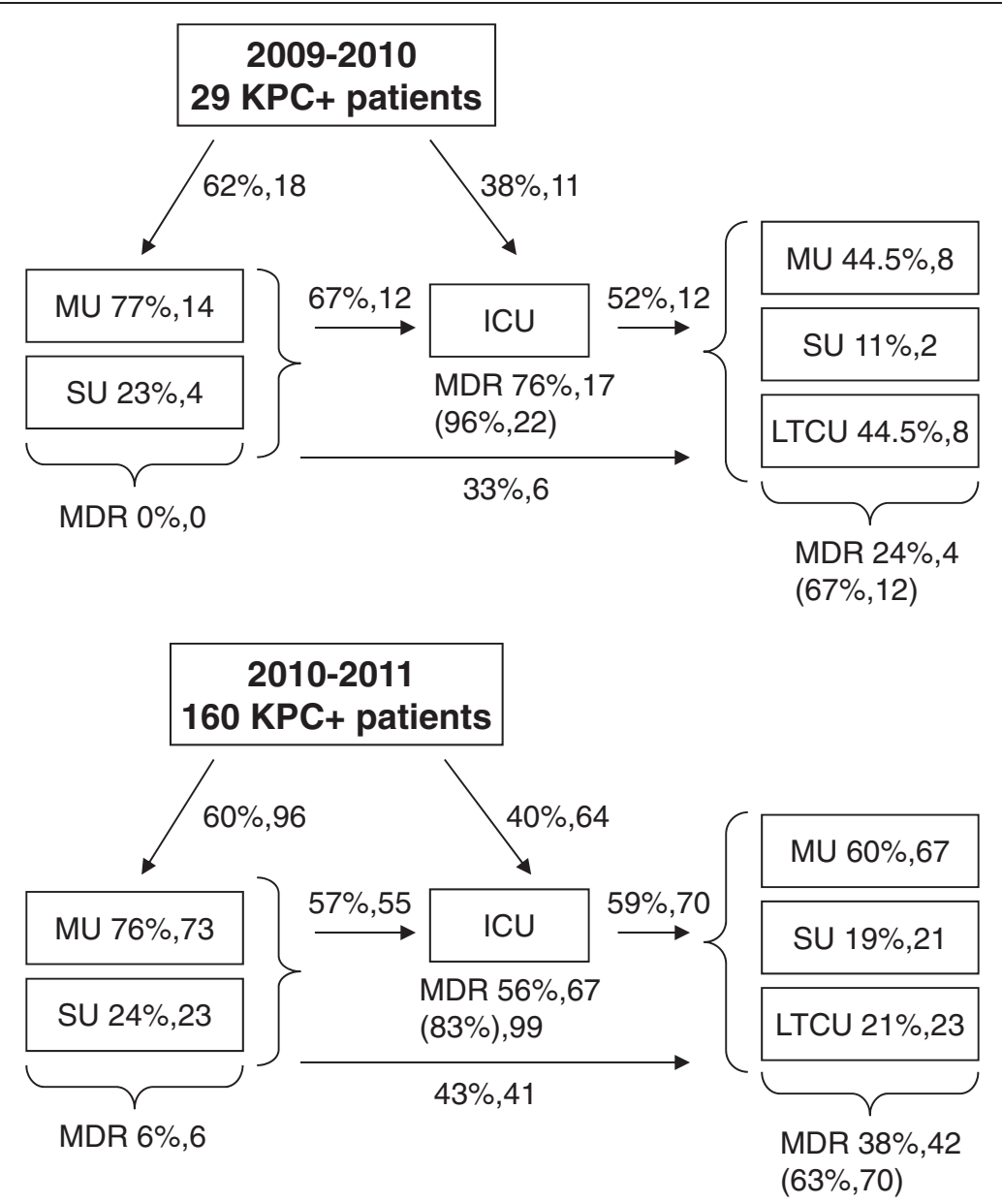

Figure 3 Relocation of KPC-positive patients among different hospital wards. Relocation has been analyzed in the two periods of time: Jan 2009-Jun 2010 and Jul 2010-Dec 2011. Percentage of MDR isolates (including KPC-positive strains) were calculated on the total number of KPC-positive patients (29 and 160 for the first and second period of analysis, respectively). The first number describes MDR percentages of patients that newly acquired MDR organisms in the indicated hospital units; the second number refers to the absolute number of patients. The third bracketed number describes the percentage of patients found with MDR isolates (both newly and previously acquired) over the total number of KPC-positive patients hospitalized in the indicated ward. Abbreviations are: ICU = Intensive Care Unit; MU = Medical Unit; SU=Surgical Unit; LTCU = Long Term Care Unit; MDR= multi-drug-resistance.

found. One KPC-3-encoding plasmid, pKpQIL [10], was detected in most of the isolates, indicating that this plasmid backbone has superior conjugation/mobility properties with respect to other identified plasmids.

In conclusion, our work showed that KPC genes have different mobility ability, some of them being very efficient in horizontal transfer within $K$. pneumoniae strains. The important consequence is that KPCmediated resistance is now present also in non-ICUs, with subsequent dangerous possible spreading to the community. This behaviour, even though detected in an Italian city, very likely reflects KPC spreading evolution worldwide. Both phenotypic and genotypic identification of carbapenemase producers, also at the carriage state, is thus urgently needed to prevent and contain further resistance spreading, especially in the case of highly transmissible plasmids.

\section{Methods}

KPC outbreak investigation and follow-up

In the city of Padua, in North-East Italy, the two main public hospitals are the Teaching Hospital (TH) and the Saint Antony Hospital (SAH), which offer 1580 and 300 beds, respectively. They are two distinct and independent hospital complexes, located around $1.5 \mathrm{~km}$ apart. Both offer clinical support in all areas of medicine. There is no routine relocation of patients, nor staff exchange between the two hospitals. Phenotypic and genotypic KPC investigation was performed from June 2009 to December 2011. Investigated clinical specimens were: 


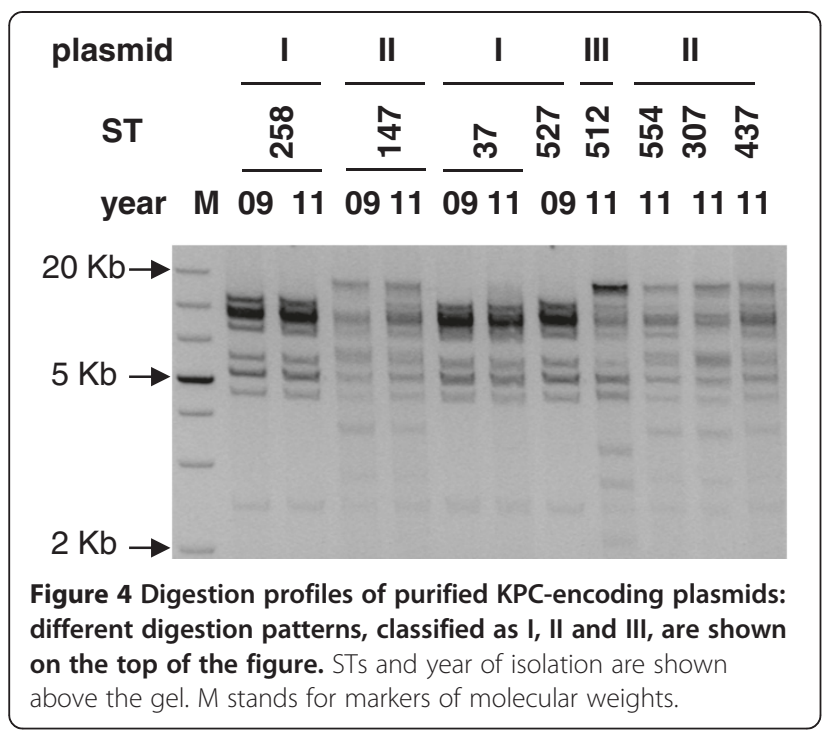

blood, urine, bronchoalveolar lavage, sputum, perirectal swab, nasal swab, wound swab, skin swab, and liquor.

\section{Clinical epidemiology}

Hospitalization data from June 2009 to December 2011 were analyzed for each patient. Considering that only few patients had been screened for KPC by genotypic methods since the beginning of their hospitalization, we assumed that KPC was acquired when the first MDR isolate (including resistance to imipenem and meropenem) was phenotypically detected. We considered this a reliable assumption because we had observed that all $K$. pneumoniae phenotypically resistant to imipenem and meropenem encoded the KPC enzyme. Moreover, patients were considered to have been newly infected/colonized with MDR organisms when at least two samples obtained from the same unit and from the same type of clinical sample had been previously found negative. Patients with at least one KPC-positive sample in a different ward or in a different type of sample were considered to have been previously infected/colonized, so that for each patient no more than one new acquisition was counted. Definitions: "KPC-positive K. pneumoniae" refers to strains that were genotypically confirmed to encode the KPC enzyme; "carbapenem-resistance" indicates resistance to carbapenem antibiotics and may be encoded by KPC or other carbapenemases; "multi-drug-resistant (MDR) organism" refers to bacteria that were phenotypically found to be resistant to all major classes of antibiotics, including carbapenems.

\section{Antimicrobial susceptibility testing and carbapenemase screening}

Clinical specimens were streaked on non-selective enrichment agar plates (BBL Blood Agar or BBL Chocolate
II Agar), and on differential and selective MacConkey II Agar plates (all from Becton Dickinson Italia, Milan, Italy), incubated at $35{ }^{\circ} \mathrm{C} \pm 2$ for $16-18 \mathrm{~h}$; if necessary, single colonies (usually, one ore two) of each colony type were further streaked on enrichment agar plates to obtain pure cultures. Microbial identification and antibiotic susceptibility testing was performed using a Vitek2 automated system (bioMerieux, Marcy l'Etoile, France). Resistance was defined using EUCAST criteria (http://www. eucast.org/fileadmin/src/media/PDFs/EUCAST_files/ Breakpoint_tables/Breakpoint_table_v_2.0_120221.pdf). The MICs of imipenem and meropenem were determined by the Etest (AB Biodisk, Solna, Sweden). The modified Hodge test was performed to detect carbapenemase production [18].

\section{PCR for detection of antibiotic resistance genes}

Resistance genes $\left(b l a_{\mathrm{KPC}}, b l a_{\mathrm{TEM}}, b l a_{\mathrm{SHV}}, b l a_{\mathrm{CTX}-\mathrm{M}}, b l a_{\mathrm{VIM}}\right.$, bla $_{\mathrm{IMP}}$ bla $a_{\mathrm{NMC}-\mathrm{IMI}}, b l a_{\mathrm{SME}}, b l a_{\mathrm{SPM}}, b l a_{\mathrm{OXA}-1}, b l a_{\mathrm{OXA}-9}$, $b l a_{\text {OXA-48 }}, b l a_{\text {OXA-58 }}, b l a_{\text {OXA-23 }}, b l a_{\text {OXA-24 }}, b l a_{\text {OXA-51 }}$, bla $\left.a_{\mathrm{OXA}-143}\right)$ were detected by PCR and sequencing $[19,20]$. Positive PCR products were sequenced with an ABI3730 sequencer (Applied Biosystems) and sequences compared with those from GenBank (www.ncbi.nlm.nih.gov/blast/).

\section{Molecular genotyping}

The genetic relatedness of carbapenem-resistant strains was determined by multilocus sequence typing (MLST), pulsed-field gel electrophoresis (PFGE) analysis and enterobacterial repetitive intergenic consensus-PCR (ERICPCR) [21, 22]. MLST was performed according to the MLST website (http://www.pasteur.fr/recherche/genopole/PF8/mlst/Kpneumoniae.html). For PFGE, total DNA extracted from bacterial cells was digested with XbaI (Fermentas Life Science), run on agarose gel with CHEF$\mathrm{DR}^{\circledR}$ III Variable Angle System (Bio-Rad Laboratories, Milan, Italy) and stained with ethidium bromide. PFGE profiles were interpreted according to Tenover criteria [23]. Isolates with the same pulsotype were classified as a clone. ERIC-PCR was performed using reported primers ERIC1 and ERIC2 [20].

\section{Plasmid analysis and transformation}

Plasmid DNA was purified using phenol/chloroform extraction and electroporated (Bio-Rad Gene Pulser ${ }^{\circledR}$, Bio-Rad Laboratories, Milan, Italy) into E. coli Top10 (Invitrogen Ltd., Paisley, United Kingdom) recipient cells. Transformed strains were selected on ampicillin agar plates $(100 \mathrm{mg} / \mathrm{L})$ and $b l a_{\mathrm{KPC}}$ presence was confirmed by PCR. Purified plasmids from transformed KPC-positive colonies were digested with EcoRI and XbaI (Fermentas Life Sciences, Milan, Italy) and run on $1 \%$ agarose gels to compare their restriction pattern. Transformants 
possessing $b l a_{\mathrm{KPC}}$ were subjected to antibiotic susceptibility testing and further molecular characterization.

\section{Plasmid sequencing}

Plasmid DNA obtained from transformed bacterial cells was sequenced by sequence walking and cloning of DNA fragments obtained by nebulization (Clonejet kit Fermentas Life Science). Nucleotide sequences were compared to the ones on the NCBI (National Center for Biotechnology Information) website. For deep sequencing, plasmid DNA extracted from samples was sequenced using a 454 FLX Sequencing Platform (Roche). Briefly, purified DNA was fragmented by nebulization, fragments in the size range of $\sim 600-900$ base pairs (bp) were selected and adaptor sequences, specific for 454 shotgun sequencing protocol, were ligated to both fragments ends. Genome libraries prepared from each isolate were uniquely bar-coded to allow multiplexing and subsequent in silico deconvolution. We obtained a total of 103914 reads from a pool of 8 samples, with a mean length of $436 \mathrm{bp}$. Assembly of sequences was conducted with Newbler 2.6 (Roche).

\section{Abbreviations}

KPC: Klebsiella pneumoniae carbapenemase; TH: Teaching Hospital; SAH: Saint Antony Hospital; ICU: Intensive care unit; ST: Sequence type; MLST: multi locus sequence typing; PFGE: pulse field gel electrophoresis;

ERIC: Enterobcterial Repetitive Intergenic Consensus; MIC: minimum inhibitory concentration; MDR: multi drug resistance.

\section{Competing interests}

The authors declare no competing interests.

\section{Acknowledgments}

We thank Dr. A. Grossato and Dr. S. Toppo for assistance on PFGE and sequencing data analysis, respectively. This work was supported by the Italian Ministry of University and Research (MIUR), Grant \# FIRB-Ideas RBID082ATK, and by the University of Padua.

\section{Author's contributions}

SNR conceived of the study, participated in its design and coordination and drafted the manuscript; IF carried out the genotypic assays; EF carried out the MLST analysis and participated in the deep sequencing analysis; CB participated in the phenotypic analysis; EL carried out the deep sequencing data analysis; LB participated in the deep sequencing analysis; AC conceived of the study and participated in its design and coordination; GP participated in the study coordination. All authors read and approved the final manuscript.

Received: 30 May 2012 Accepted: 16 July 2012

Published: 16 July 2012

\section{References}

1. Nordmann P, Cuzon G, Naas T: The real threat of Klebsiella pneumoniae carbapenemase-producing bacteria. Lancet Infect Dis 2009, 9:228-236.

2. Poirel L, Pitout JD, Nordmann P: Carbapenemases: molecular diversity and clinical consequences. Future Microbiol 2007, 2:501-512.

3. Munoz-Price LS, Quinn JP: The spread of Klebsiella pneumoniae carbapenemases: a tale of strains, plasmids, and transposons. Clin Infect Dis 2009, 49:1739-1741.

4. Pitout JD, Laupland KB: Extended-spectrum beta-lactamase-producing Enterobacteriaceae: an emerging public-health concern. Lancet Infect Dis 2008, 8:159-166.
5. Yigit H, Queenan AM, Anderson GJ, Domenech-Sanchez A, Biddle JW, Steward CD, Alberti S, Bush K, Tenover FC: Novel carbapenem-hydrolyzing beta-lactamase, KPC-1, from a carbapenem-resistant strain of Klebsiella pneumoniae. Antimicrob Agents Chemother 2001, 45:1151-1161.

6. Samra Z, Ofir O, Lishtzinsky Y, Madar-Shapiro L, Bishara J: Outbreak of carbapenem-resistant Klebsiella pneumoniae producing KPC-3 in a tertiary medical centre in Israel. Int J Antimicrob Agents 2007, 30:525-529.

7. Giakkoupi P, Papagiannitsis CC, Miriagou V, Pappa O, Polemis M, Tryfinopoulou K, Tzouvelekis LS, Vatopoulos AC: An update of the evolving epidemic of blaKPC-2-carrying Klebsiella pneumoniae in Greece (2009-10). J Antimicrob Chemother 2011, 66:1510-1513.

8. Gaibani P, Ambretti S, Berlingeri A, Gelsomino F, Bielli A, Landini MP, Sambri $\checkmark$ : Rapid increase of carbapenemase-producing Klebsiella pneumoniae strains in a large Italian hospital: surveillance period 1 March - 30 September 2010. Euro Surveill 2011, 16(8):-

9. Bush K: Alarming beta-lactamase-mediated resistance in multidrugresistant Enterobacteriaceae. Curr Opin Microbiol 2010, 13:558-564

10. Leavitt A, Chmelnitsky I, Carmeli Y, Navon-Venezia S: Complete nucleotide sequence of KPC-3-encoding plasmid pKpQIL in the epidemic Klebsiella pneumoniae sequence type 258. Antimicrob Agents Chemother 2010, 54:4493-4496.

11. Hirsch EB, Tam VH: Detection and treatment options for Klebsiella pneumoniae carbapenemases (KPCs): an emerging cause of multidrug-resistant infection. J Antimicrob Chemother 2010, 65:1119-1125.

12. Kitchel B, Rasheed JK, Patel JB, Srinivasan A, Navon-Venezia S, Carmeli Y, Brolund A, Giske CG: Molecular epidemiology of KPC-producing Klebsiella pneumoniae isolates in the United States: clonal expansion of multilocus sequence type 258. Antimicrob Agents Chemother 2009, 53:3365-3370.

13. Garcia-Fernandez A, Villa L, Carta C, Venditti C, Giordano A, Venditti M, Mancini C, Carattoli A: Klebsiella pneumoniae ST258 producing KPC-3 identified in italy carries novel plasmids and OmpK36/OmpK35 porin variants. Antimicrob Agents Chemother 2012, 56:2143-2145.

14. Samuelsen $O$, Naseer U, Tofteland S, Skutlaberg DH, Onken A, Hjetland R, Sundsfjord A, Giske CG: Emergence of clonally related Klebsiella pneumoniae isolates of sequence type 258 producing plasmid-mediated KPC carbapenemase in Norway and Sweden. J Antimicrob Chemother 2009, 63:654-658.

15. Endimiani A, Hujer AM, Perez F, Bethel CR, Hujer KM, Kroeger J, Oethinger M, Paterson DL, Adams MD, Jacobs MR, et al: Characterization of blaKPC-containing Klebsiella pneumoniae isolates detected in different institutions in the Eastern USA. J Antimicrob Chemother 2009, 63:427-437.

16. Giakkoupi P, Pappa O, Polemis M, Vatopoulos AC, Miriagou V, Zioga A, Papagiannitsis CC, Tzouvelekis LS: Emerging Klebsiella pneumoniae isolates coproducing KPC-2 and VIM-1 carbapenemases. Antimicrob Agents Chemother 2009, 53:4048-4050.

17. Steinmann J, Kaase M, Gatermann S, Popp W, Steinmann E, Damman M, Paul A, Saner F, Buer J, Rath P: Outbreak due to a Klebsiella pneumoniae strain harbouring KPC-2 and VIM-1 in a German university hospital, July 2010 to January 2011. Euro Surveill 2011, 16(33):-

18. Girlich D, Poirel L, Nordmann P: Value of the modified Hodge test for detection of emerging carbapenemases in Enterobacteriaceae. J Clin Microbiol 2012, 50:477-479.

19. Kaczmarek FM, Dib-Hajj F, Shang W, Gootz TD: High-level carbapenem resistance in a Klebsiella pneumoniae clinical isolate is due to the combination of bla(ACT-1) beta-lactamase production, porin OmpK35/36 insertional inactivation, and down-regulation of the phosphate transport porin phoe. Antimicrob Agents Chemother 2006, 50:3396-3406.

20. Richter SN, Frasson I, Bergo C, Manganelli R, Cavallaro A, Palu G: Characterisation of qnr plasmid-mediated quinolone resistance in Enterobacteriaceae from Italy: association of the qnrB19 allele with the integron element ISCR1 in Escherichia coli. Int J Antimicrob Agents 2010, 35:578-583.

21. Lavilla S, Gonzalez-Lopez JJ, Sabate M, Garcia-Fernandez A, Larrosa MN Bartolome RM, Carattoli A, Prats G: Prevalence of qnr genes among extended-spectrum beta-lactamase-producing enterobacterial isolates in Barcelona, Spain. J Antimicrob Chemother 2008, 61:291-295.

22. Kidd TJ, Grimwood K, Ramsay KA, Rainey PB, Bell SC: Comparison of three molecular techniques for typing Pseudomonas aeruginosa isolates in sputum samples from patients with cystic fibrosis. J Clin Microbiol 2012, 49:263-268. 
23. Tenover FC, Arbeit RD, Goering RV, Mickelsen PA, Murray BE, Persing DH, Swaminathan B: Interpreting chromosomal DNA restriction patterns produced by pulsed-field gel electrophoresis: criteria for bacterial strain typing. J Clin Microbiol 1995, 33:2233-2239.

doi:10.1186/1757-4749-4-7

Cite this article as: Richter et al: KPC-mediated resistance in Klebsiella pneumoniae in two hospitals in Padua, Italy, June

2009-December 2011: massive spreading of a KPC-3-encoding plasmid and involvement of non-intensive care units. Gut Pathogens 2012 4:7.

\section{Submit your next manuscript to BioMed Central and take full advantage of:}

- Convenient online submission

- Thorough peer review

- No space constraints or color figure charges

- Immediate publication on acceptance

- Inclusion in PubMed, CAS, Scopus and Google Scholar

- Research which is freely available for redistribution 